\title{
Study on the Dose-Effect Relationship of Selenite with the Growth of Wheat
}

\author{
A. Peng, * Y. Xu, J. H. LiU, and Z. J. Wang \\ SKLEAC, Research Center for Eco-environmental Sciences, \\ Academia Sinica, Beijing, 100085, People's Republic of China
}

Received November 5, 1999; Accepted January 5, 2000

\begin{abstract}
The wheat in hydroponics culture has been chosen as a model to study the dose-effect relationship of selenite with its growth. Five different morphological end points and 12 different biological and biochemical end points during different phases of growth of wheat seedling and seed germination have been measured and analyzed. A dose-effect relationship of selenite with the growth of wheat has been obtained from data analysis. The results shows that critical threshold for beneficial effect is about $1.0 \mathrm{mg} \mathrm{Se} / \mathrm{L}$, and the critical threshold for adverse effect is about $5.0 \mathrm{mg} \mathrm{Se} / \mathrm{L}$ in hydroponics culture. From the result of product of lipid peroxidation, it is assumed that the role of selenium for plant is related to the oxy-radical reaction.
\end{abstract}

Index Entries: Selenium; dose-effect relationship; wheat; hydroponic culture; toxic effect; critical threshold.

\section{INTRODUCTION}

Selenium is an essential element for animal growth. However, it can also be toxic at higher concentrations. The safety amount of intake for human has been determined (1). However, it is unclear whether selenium is an essential element for plants which are key links of food chain. A number of nutritional and toxic properties of selenium on plants have been found (2). For example, it has been shown that a small amount of Se could stimulate the growth of an Se-accumulating plant,

*Author to whom all correspondence and reprint requests should be addressed. 
whereas these same concentrations were highly inhibitory to the growth of non-Se-accumulating plants (3). Another study demonstrated that an adequate amount of Se could accelerate growth of rice and raise its yield (4). These studies have deepened our understanding of the function of selenium in plants. Nevertheless, a systematic analysis of the dose-response relationship of Se with the growth of grain has not been conducted.

In this study, wheat in hydroponics culture was chosen as an experimental model, and 5 end points about morphology and 12 end points about biology and biochemistry during different phases of growth of wheat seedling and germination have been measured. From data analysis, a dose-effect relationship of selenite with the growth of wheat has been obtained.

\section{MATERIAL AND METHODS}

\section{Incubation}

The hydroponics culture of wheat was chosen as an experimental model to assay the end points of seed germination and growth of wheat. The method of seedlings experiments are the same as described in our previous reports $(5,6)$.

\section{Assay Methods}

The physiological measurements, including germination rate, bud length, fresh weight, root length, and biomass, were measured. The method and references for determination of various items are listed in Table 1.

\section{Data Treatment}

The dose-effect relationship of selenite was obtained using statistical and interrelative analyses of the physiological and biochemical end points. The control group without Se was used for comparison. The determination of either the positive or negative effect of the Se dosage was set at $10 \%$ higher or lower compared to those of the control group, respectively. The effect was considered significant if the end point was more than $25 \%$ of the control group. A significant positive effect indicates stimulation of wheat growth (aptimum concentration), whereas a significant negative effect shows toxic inhibition (significant toxic concentration). 
Table 1

The Method for Biochemical

and Physiological Assay

\begin{tabular}{ll}
\hline Items & Author \\
\hline Seed vigor & Steponkus P. L. (7) \\
Activity of a -arnylase & Miller G. L. (8) \\
Activity of B -amylase & Miller G. L. (8) \\
Chrolophyll A & Arron D. I. (9) \\
Chlorophyll B & Arron D. I. (9) \\
Chlorophyll A+B & Arron D. I. (9) \\
Chlorophyll K & Arron D. I. (9) \\
Membrane permeability & Liu H.X. (10) \\
Free Proline & Bates L. S. (11) \\
Protein content & Brodford M. M. (12) \\
MDA & Dhinds R. S. (13) \\
Activity of POD & Zhu Z. C. (14) \\
POD isozyme spectrum & Davis B. J. (15) \\
Sugar content & Handbook (16) \\
\hline
\end{tabular}

\section{RESULTS AND DISCUSSION}

The morphological changes of wheat during different phases of growth of wheat seedling and seed germination have been observed. The results showed that the morphological change of a greater than 5-mg Se/L treatment were obvious: the stems became short and small, the leaves grew slender, and the tip of the leaves looked yellowish, indicating a lack of chlorophyll. Several exert data have been reported in our previous article (6). For example, selenite under the concentration level of $1.0 \mathrm{mg} / \mathrm{L}$ stimulated the development of bud at $3 \mathrm{~d}$ and the turnover value decreased to $0.1 \mathrm{mg} / \mathrm{L}$ at $5 \mathrm{~d}$. When a higher selenite concentration (>5.0 mg/L) was used, selenite significantly inhibited bud development. The results of data treatment indicated that at significant levels, the inhibitory threshold of selenite for the germination rate was $5 \mathrm{mg} / \mathrm{L}(p<0.01)$, the seed vigor was stimulated when the concentration of selenite was below $0.5 \mathrm{mg} \mathrm{Se} / \mathrm{L}(p<0.001)$, the growth of roots was stimulated at Se concentration of $<1.0 \mathrm{mg} / \mathrm{L}$, a sharp decrease of fresh weight of the seedling was observed in the 5-mg Se/L treatment, the enzymatic activity of $\alpha$-amylase was found to be the highest at $0.1 \mathrm{mg} \mathrm{Se} / \mathrm{L}$ $(p<0.001)$, decreasing with the increase of Se dosage exceeding $10 \mathrm{mg}$ $\mathrm{Se} / \mathrm{L}$, and the inhibition effect of selenite for the synthesis of chlorophyll was $>1 \mathrm{mg} / \mathrm{L}$. Based on the data from both biological and biochemical measurements, the toxic threshold of selenite for wheat in a hydroponic culture varied with the indicator used. It varies from 1 to $5 \mathrm{mg} \mathrm{Se} / \mathrm{L}$ and 
Table 2

The Critical Threshold of Selenite Content in Wheat

\begin{tabular}{l|cccc}
\hline \multirow{2}{*}{ Items } & \multicolumn{4}{|c}{ Threshold for Se (mg/L) } \\
\cline { 2 - 5 } & Benefitial dose & Optimium dose & Adverse dose & Toxic dose \\
\hline Germination rate & $\sim 1.0$ & 0.5 & $>1.0$ & 20.0 \\
Seed vigor & $\sim 1.0$ & 0.1 & $>1.0$ & 5.0 \\
Bud length & $\sim 1.0$ & 0.5 & $>1.0$ & 10.0 \\
Seedling fresh weight & $\sim 1.0$ & 0.1 & $>1.0$ & 5.0 \\
Seedling height & $\sim 1.0$ & 0.1 & $>1.0$ & 10.0 \\
Membrane permeablity & $\sim 1.0$ & 0.1 & $>1.0$ & 10.0 \\
Free proline & $\sim 1.0$ & 0.1 & $>1.0$ & 5.0 \\
Chrolophyll A & $\sim 1.0$ & 0.1 & $>1.0$ & $10 . \hat{2}$ \\
Chrolophyll B & $\sim 1.0$ & 0.1 & $>1.0$ & 10.0 \\
Chrolophyll A+B & $\sim 1.0$ & 0.1 & $>1.0$ & 10.0 \\
Chrolophyll K & $\sim 1.0$ & 0.1 & $>1.0$ & 10.0 \\
Sugar content & $<0.5$ & 0.1 & $>1.0$ & 5.0 \\
Activity a -amylase & $\sim 1.0$ & 0.1 & $>0.5$ & 5.0 \\
Activity $\beta$-amyase & $\sim 1.0$ & 0.5 & $>1.0$ & 20.0 \\
Protein conten & -- & 0.1 &.- & - \\
Malonic dialdehyde & $\sim 1.0$ & 0.1 & $>1.0$ & 5.0 \\
Activity of POD & $\sim 1.0$ & 0.5 & $>1.0$ & 10.0 \\
\hline
\end{tabular}

is reflected most sensitively in seed vigor, plumula fresh weight, chlorophyll $\mathrm{A}$ and $\mathrm{K}$, and free-proline content in leaf.

The critical threshold of selenite of the six end points for morphology and the 15 end points for physiology and biochemistry with different phases of growth of wheat seedling and seed germination are summarized in Table 2.

Through the data treatment, the scheme of the dose-biological effect relationship of selenite is shown in Fig. 1.

The ordinate is a comprehensive effect of selenite in Fig. 1, and the "plateau" of the curve shows the better nutrition concentration of Se. The effects go down when the concentration of selenite is $>1.0 \mathrm{mg} / \mathrm{L}$, marked dropping at $>5 \mathrm{mg} / \mathrm{L}$. It can be seen that seed germination and growth of wheat were stimulated by selenite in the $0.1-$ to $1.0-\mathrm{mg} / \mathrm{L}$ range; when the concentration of selenite exceeded $1.0 \mathrm{mg} / \mathrm{L}$, toxic effects were obtained, and greater than $5.0 \mathrm{mg} / \mathrm{L}$, significant toxic effects were observed. Se accumulators represent evidence that Se may be a possible micronutrient. It has been accepted Se is an essential element for animals, but it has not been widely accepted in such a role for plants. The result of this experiment may sustain this assumption. It is known 


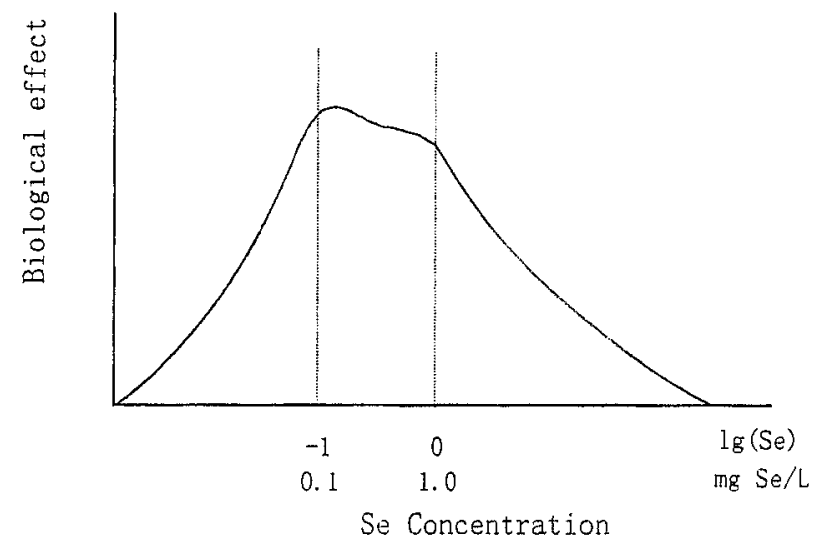

Fig. 1. The plot of the dose-biological effect relationship for selenite.

that trace selenium play an important role in oxygen metabolism and, therefore, in the formation of free radicals. Based on the results in vivo and in vitro experiments, Zhou et al. have indicated that relationships between the concentration of selenium with both the level of free radicals and its biological effects were directly in keeping with the Bertrand rule, and the former may be one of the mechanisms for the dose-effect relationship of selenium to the latter (17). It was assumed that at a lower concentration of selenite, Se was a protective factor and freeradical scavenger, but at a higher concentration, Se could be generated as an oxy-radical because of oxidative stress and could damage the cell membrane.

To learn more about the mechanism of the stimulation and inhibition of selenite in plants, the product of lipid peroxidation and malonic dialdehyde (MDA) has been measured in wheat seedling after at culture of 1 and 2 wk. The results are shown in Fig. 2.

The trend of curves A and B in Fig. 2 is similar. A marked decrease of the content of MDA when the selenite concentration increased from the check (CK) group to $0.1 \mathrm{Se} \mathrm{mg} / \mathrm{L}$ is seen, and there is a progressive increase when the selenite concentration increased slightly. For 1.0 Se $\mathrm{mg} / \mathrm{L}$, the increase of MDA was responsible for the variation of peroxidative stress brought about by the increasing selenite concentration in the medium. These results obtained from hydroponic culture identify that the concentration of Se and its biological activity is related to the oxy-radical reaction and corresponds to Huang's results in the barley test, from which it was assumed that Se is required for biosynthesis of the selenoperoxidase GPX (18), which can play an important role in cytoprotection against oxidative damage. 


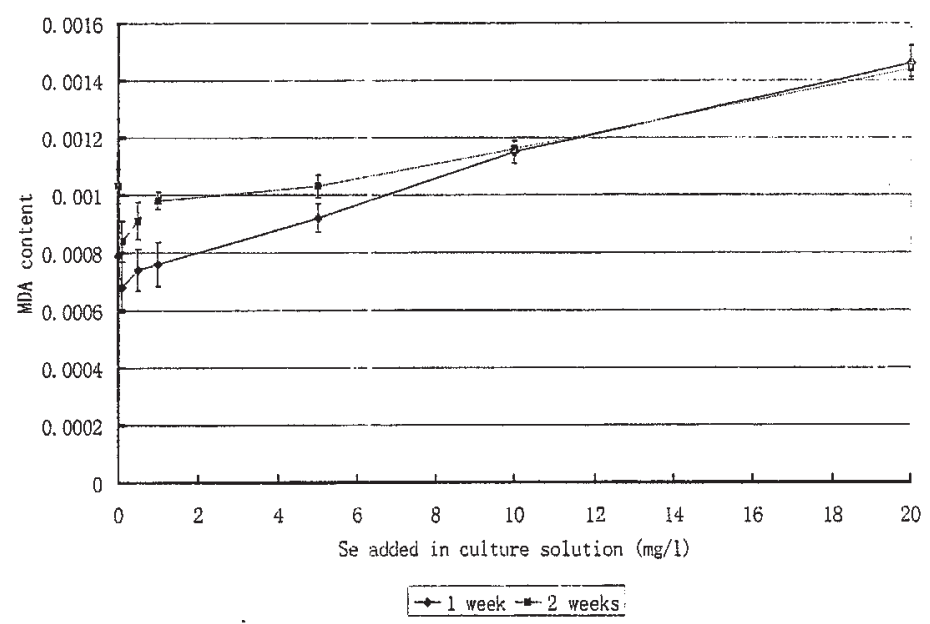

Fig. 2. Influence of $\mathrm{Na}_{2} \mathrm{SeO}_{3}$ on $\mathrm{MDA}$ content of wheat. (The units for MDA content are $\mathrm{nmol} / \mathrm{g} \cdot \mathrm{FW}$.)

\section{CONCLUSIONS}

A dose-effect relationship of selenite with growth of wheat seedling and seed generated in hydroponics culture has been obtained from the physiological and biochemical studies and the data were analyzed. It was found that the critical threshold for beneficial effect is approx $1.0 \mathrm{mg}$ $\mathrm{Se} / \mathrm{L}$, and the critical threshold for adverse effect was approx $5.0 \mathrm{mg}$ $\mathrm{Se} / \mathrm{L}$ in a hydroponics culture. From the result of the product of lipid peroxidation, it is suggested that the role of selenite for plant is closely related to the oxy-radical reaction.

\section{ACKNOWLEDGMENTS}

This work was supported by a grant from the Chinese National Science Foundation No. 29677024.

\section{REFERENCES}

1. G. Q. Yang, Studies of human selenium requirements in China, in G. F. Combs, O. A. Levander, J. E. Spallholz, and J. E. Oldfield, eds. Selenium in Biology and Medicine, 3th Symposium, Van Nostrand Reinhold, New York, pp. 589-618 (1987).

2. T. C. Broyer, O. C. Lee, and C. J. Asher, Selenium nutrition of green plants, effect of selenite supply on growth and selenium content of alfalfa and subterranean clover, Plant Physiol. 44, 1425-1428 (1966).

3. S. F. Trelease and H. M. Trelease, Selenium as a stimulating and possible essential element for certain plants, Science 15, 57-58 (1990).

4. M. H. Zhou, Influence of selenium on growth, root vigor and seed morphology of rice, Gui-Zhou Agri. Sci. (China) 15, 57-59 (1990). 
5. Y. Xu, Z. J. Wang, and A. Peng, Effect of fulvic acid in environment on the uptake selenium of wheat, J. Ecol. (China) 16, 135-139 (1996).

6. Z. J. Wang, Y. Xu, and A. Peng, Influences of fulvic acid on bioavailability and toxicity of selenite for wheat seedling and growth, Biol. Trace Element Res. 55, 147-162 (1996).

7. P. L. Steponkus and F. O. Lanphear, Refinement of the triphenyl tetrazolium chloride method of determining cold injury, Plant Physiol. 42, 1423-1426 (1967).

8. G. L. Miller, Use of dinitro-salicylic acid reagent for determination of reducing sugar, Anal. Chem. 31, 426-428 (1959).

9. D. I. Arnon, Copper enzymes in isolated chloroplants polyphenloxidase in Beet Vulgaris, Plant Physiol. 24, 1-4 (1949).

10. H. X. Liu, S. X. Zeng, Y. R. Wang, P. Li, D. F. Chen, and J. Y. Guo, Effect of low temperature on peroxidase activity of cucumbers seedling leaf with various ability of cold-resisting, Acta Plant Physiol. (China) 11, 48-57 (1985).

11. L. S. Bates, R. P. Waldren, and I. D. Teare, Rapid determination of free proline for water stress studies, Plant Soil 39, 205-214 (1973).

12. M. M. Bradford, A rapid and sensitive method for the quantities of microgram quantitative of protein utilizing the principle of protein dries binding, Anal. Biochem. 72, 248-251 (1976).

13. R. S. Dhindsa, P. D. Pamela, and T. A. Thorpe, Leaf senescence: correlated with increased levels of membrane permeability and lipid peroxidation and decrease levels of superoxide dismutase and catalase, J. Exp. Bot. 32, 93-95 (1981).

14. C. Z. Zhu, Determination of activity of peroxidase, Adv. Biochem. Biophys. (China) 6, 80-83 (1985).

15. B. J. Davis, Disc electrophoresis: Method and application to human serum protein, Ann. NY Acad. Sci. 121, 404-407 (1964).

16. Shanghai Plant Physiology Committee, Experimental Handbook of Plant Physiology, Shanghai Science and Technology Press, Shanghai, pp. 67-70 (1981).

17. J. Y. Zhou, B. H. Chen, and H. B. Xu, The dose-effect of selenium and active oxygen species, The Sixth International Symposium on Selenium in Biology and Medicine, Abstract Book, Beijing, p. 130 (1996).

18. K. X. Huang, Study on containing-selenium bio-macromolecule in plant, Ph.D. thesis, Hua-Zhong Polytechnical University, Wuhan, China, pp. 27-29 (1995). 